\title{
Conserving slave boson approximations for the Anderson model beyond NCA
}

\author{
J. Kroha ${ }^{a * \dagger}$, P. Wölfle, ${ }^{a \dagger}$ T. A. Costi, ${ }^{a \dagger}$, P. J. Hirschfeld ${ }^{b}$ and K. A. Muttalib ${ }^{b \dagger}$
}

${ }^{a}$ Inst. f. Theorie der Kondensierten Materie, Universität Karlsruhe, Postf. 6980, 76128 Karlsruhe, Germany

${ }^{b}$ Department of Physics, University of Florida, P.O. Box 118440, Gainesville, FL 32611, U.S.A.

We derive a general scheme to construct conserving slave boson approximations for the single-impurity Anderson model beyond the noncrossing approximation (NCA). The pseudofermion and slave boson spectral functions are computed in a conserving $\mathrm{T}$-matrix approximation which includes the maximum number of impurity spin flips in each order of the hybridization. In a perturbative evaluation, the singlet channel of the conduction electron-pseudofermion $\mathrm{T}$-matrix has a pole which is renormalized by selfconsistency. As a result, the exponents of the infra-red powerlaw behavior of the pseudoparticle spectral functions are modified w.r.t. NCA and depend on the impurity occupation number. We present results for the exponents in the Kondo regime which are consistent with exact values given by the $\mathrm{x}$-ray emission and absorption exponents.

The Anderson model of an electron in a local impurity orbital hybridizing with a band of conduction electrons $c$ may be regarded as the simplest model that incorporates the complications arising from the strong onsite Coulomb repulsion $U$ which lies at the heart of strongly correlated lattice fermion models like the Hubbard, the $t-J$ and the periodic Anderson models. These are expected to describe heavy fermion systems and possibly high $T_{c}$ superconductors. Thus, the single-impurity Anderson model may serve as a test ground for theoretical techniques for strongly correlated electron systems.

The strong repulsion $U$ effectively limits the dynamics of the electrons to the part of the Hilbert space with no double occupancy of the impurity level. This may be implemented exactly [1] by separating the impurity electron operator $d_{\sigma}$ into two auxiliary fields $f_{\sigma}, b$ which carry spin $\sigma$ and the local charge, respectively, $d_{\sigma}^{+}=f_{\sigma}^{+} b$, and which are subject to the local operator constraint $\hat{Q}=$ $\sum_{\sigma} f_{\sigma}^{+} f_{\sigma}+b^{+} b=1$. The projection onto the $\hat{Q}=1$ Hilbert subspace produces an orthogonality catastrophe [2] which leads to a threshold behavior and to infrared powerlaw divergences of the auxiliary particle spectral densities with exponents $\alpha_{f}$ and $\alpha_{b}$, respectively. Special interest in the auxiliary particle dynamics has been inspired by the recent discovery [3] that in the Kondo limit of the multi-channel Anderson models, which exhibit overcomplete spin

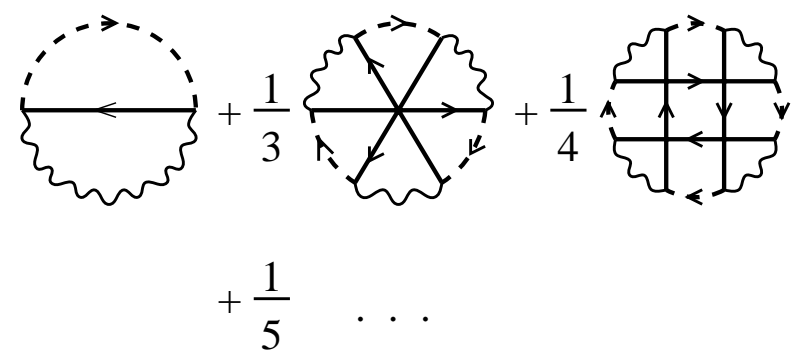

Figure 1: Infinite series of free energy diagrams generating the STMA. Solid, dashed and wiggly lines correspond to the local conduction electron, the pseudofermion and the slave boson propagators, respectively.

screening and a non-Fermi liquid ground state, the low-frequency singular behavior of the $f$ and $b$ propagators is correctly described by a mean field theory. This may be interpreted as a separation of the spin and charge degrees of freedom. On the other hand, for the single-channel spin $1 / 2$ Anderson impurity model (spin degeneracy $N=2$ ), the pseudofermion and the slave boson degrees of freedom $f, b$ must recombine at temperatures $T$ below the Kondo scale $T_{K}$ in order to form the Fermi liquid ground state

* Work performed in part at Cornell University, USA; supported by the A. v. Humboldt Foundation. $\dagger$ Supported by SFB 195 of the DFG. 


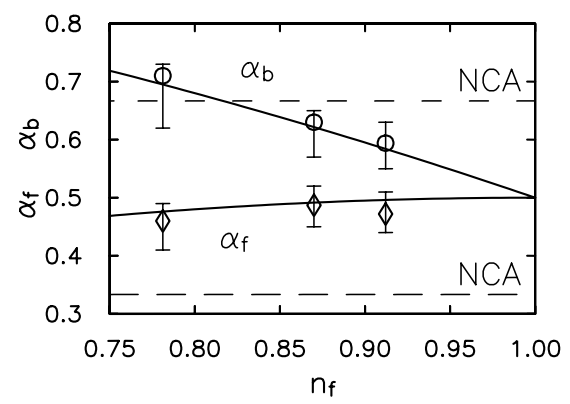

Figure 2: Auxiliary particle exponents $\alpha_{f}, \alpha_{b}$ in the Kondo regime as a function of $n_{f}$. Data points with error bars represent the STMA results, consistent with the $\mathrm{x}$-ray emission and absorption exponents (solid lines), respectively. Dashed lines: NCA results.

known [4] to be characteristic of this model. It is the reconstruction of the physical electron propagator $G_{d \sigma}(\omega)$ from the auxiliary particle propagators $G_{f \sigma}(\omega)$ and $G_{b}(\omega)$, which is difficult to achieve such that the Fermi liquid relations are satisfied [5]. The leading order selfconsistent approximation in $1 / N$ or in the hybridization $V$, the NCA, although qualitatively correct at higher $T$, produces spurious singularities in the Fermi liquid regime [1]. A general scheme of constructing controlled slave boson approximations is implied by the requirement that, in addition to the exact projection onto the $\hat{Q}=1$ subspace, the two U(1) gauge symmetries of the Anderson Hamiltonian associated with the conservation of the local pseudoparticle number, $n_{f}+n_{b}=1$, and of the total fermion number, $n_{c \sigma}+n_{f \sigma}=$ const, must be preserved. Hence, the selfenergies of the pseudofermions, the slave bosons and the local conduction electrons are derived from one generating functional $\Phi: \Sigma_{f \sigma}=\frac{\delta \Phi}{\delta G_{f \sigma}}, \quad \Sigma_{b}=\frac{\delta \Phi}{\delta G_{b}}, \quad \Sigma_{c \sigma}=\frac{\delta \Phi}{\delta G_{c 0 \sigma}}$. Since the projected $d$ electron propagator is given exactly by $G_{d \sigma}(\omega)=\Sigma_{c \sigma}(\omega) / V^{2}$, this also provides a prescription of how to calculate $G_{d \sigma}$ in consistence with the auxiliary propagators.

Motivated by the fact that the Fermi liquid fixed point arises due to the formation of the Kondo singlet state, we evaluate the conserving approximation generated by the $\Phi$ functional shown in Fig. 1, which consists of the infinite series of skeleton diagrams that include the maximum number of fermion spin flips at any given order in the boson propagator $G_{b}$. Note that the first diagram corresponds to the NCA. The first three diagrams include all corrections up to $O\left(1 / N^{2}\right)$. They correspond to an extension of the NCA which constitutes a significant im- provement [6], but still does not correctly describe the dynamics at energies well below $T_{K}$. The infinite summation (Fig. 1) defines a selfconsistent Tmatrix approximation (STMA) which involves the conduction electron-pseudofermion $\mathrm{T}$-matrix $T_{f c}$ in the selfenergies. Solving the linear Bethe-Salpeter equation for $T_{f c}$ in perturbation theory, i.e. taking the NCA for the single auxiliary propagators, we find for $T<T_{K}$ a pole in the singlet channel of $T_{f c}$ as a function of the $f-c$ center-of-mass frequency $\Omega$ at $\Omega$ roughly equal to $-T_{K}[7]$. The threshold behavior of all auxiliary particle functions implies that at $T=0$ the pole must necessarily lie on the real axis. It indicates that an infinite summation of terms to all orders of $1 / N$ is inevitable in order to capture this singularity, which may presumably be identified with the onset of the formation of the Kondo singlet state. We now turn to the full evaluation of the STMA. The exact property of all pseudoparticle spectral functions being 0 for negative frequencies at $T=0$ implies that the perturbative pole is shifted to $\Omega=0$ by selfconsistency, so that the two-particle $f-c$ bound state merges with the incoherent spectral weight present at $\Omega \geq 0$. As a result, the auxiliary particle threshold exponents $\alpha_{f}$, $\alpha_{b}$ are renormalized as compared to the NCA. They depend on the impurity occupation number $n_{f}$ and are in good quantitative agreement with the result of an NRG calculation [8] that they coincide with the emission and absorption exponents of the x-ray problem (Fig. 2).

\section{REFERENCES}

[1] For a review see N. E. Bickers, Rev.Mod.Phys. 59, 845 (1987) and references therein.

[2] P.W.Anderson, Phys.Rev.Lett. 18, 1049 (1967).

[3] D. L. Cox and A. E. Ruckenstein, Phys. Rev. Lett. 71, 1613 (1993).

[4] P. Schlottmann, Phys. Rep. 181, 1 (1989).

[5] T. A. Costi, J. Kroha, and P. Wölfle, Phys. Rev. B53, 1850 (1996).

[6] F. Anders and N. Grewe, Europhys. Lett. 26, 551 (1994); F. Anders, J. Phys. Cond. Mat. 7 2801 (1995).

[7] J. Kroha, Ph.D. thesis, Univ. Karlsruhe (1993); J. Kroha, P. J. Hirschfeld, K. A. Muttalib, and P. Wölfle, Solid State Comm. 83, 1003 (1992).

[8] T. A. Costi, P. Schmitteckert, J. Kroha, and P. Wölfle, Phys. Rev. Lett. 73, 1275 (1994). See also B. Menge and E. Müller-Hartmann, Z. Phys. B73, 225 (1988). 